\title{
Optimization Study on the Magnetic Field of Superconducting Halbach Array Magnet
}

\author{
Boyang Shen*, Jianzhao Geng, Chao Li, Xiuchang Zhang, Lin Fu, Heng Zhang, Jun Ma, and T. A. Coombs** \\ Electrical Engineering Division, Department of Engineering, University of Cambridge, CB3 0FA, UK
}

\begin{abstract}
This paper presents the optimization on the strength and homogeneity of magnetic field from superconducting Halbach Array magnet. Conventional Halbach Array uses a special arrangement of permanent magnets which can generate homogeneous magnetic field. Superconducting Halbach Array utilizes High Temperature Superconductor (HTS) to construct an electromagnet to work below its critical temperature, which performs equivalently to the permanent magnet based Halbach Array. The simulations of superconducting Halbach Array were carried out using $H$-formulation based on $B$ dependent critical current density and bulk approximation, with the FEM platform COMSOL Multiphysics. The optimization focused on the coils' location, as well as the geometry and numbers of coils on the premise of maintaining the total amount of superconductor. Results show Halbach Array configuration based superconducting magnet is able to generate the magnetic field with intensity over 1 Tesla and improved homogeneity using proper optimization methods. Mathematical relation of these optimization parameters with the intensity and homogeneity of magnetic field was developed.
\end{abstract}

Keywords: Superconducting magnet, Halbach Array, High Temperature Superconductor (HTS), HTS coils.

\section{Introduction}

Since 20th century, superconducting magnets have become one of the most beneficial superconducting applications, which are widely utilized in Magnetic Resonance Imaging (MRI) and other biological equipments [1]. Superconducting magnets are able to generate magnetic fields with desirable homogeneity and strength up to tens of Tesla [1].

In our previous research [2], we have proposed a compact superconducting magnet equipped in the Lorentz Force Electrical Impedance Tomography (LFEIT), which used the Halbach array configuration for the electromagnetic magnet $[2,3]$. Halbach array is generally permanent magnet based due to the low cost and easy maintenance [4]. However, if Halbach magnet requires intensity more than 1 Tesla $(\mathrm{T})$ which is necessary for a large-scale whole body scanner diagnostic LFEIT system, permanent magnets are not able to realise the portable functionality of LFEIT system due to their heavy

\footnotetext{
* Corresponding author. Tel.: +44 7869688318.

** Corresponding author. Tel.: +44 1223748315.

E-mail addresses: bs506@cam.ac.uk (B. Shen),jg717@cam.ac.uk (J. Geng), c1644@cam.ac.uk (C. Li), xz326@cam.ac.uk (X. Zhang), 1f359@cam.ac.uk (L. Fu), hz301@cam.ac.uk (H. Zhang), jm2104@cam.ac.uk (J. Ma), tac1000@cam.ac.uk (T.A. Coombs).
}

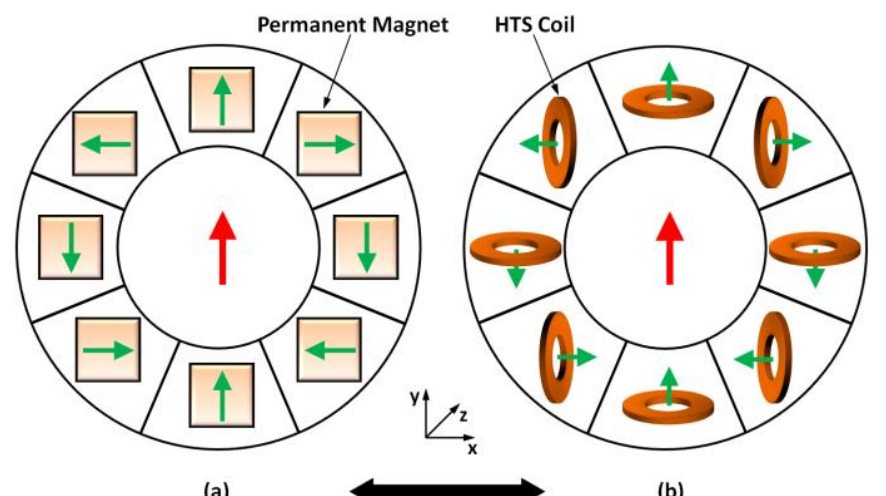

(a)

(b)

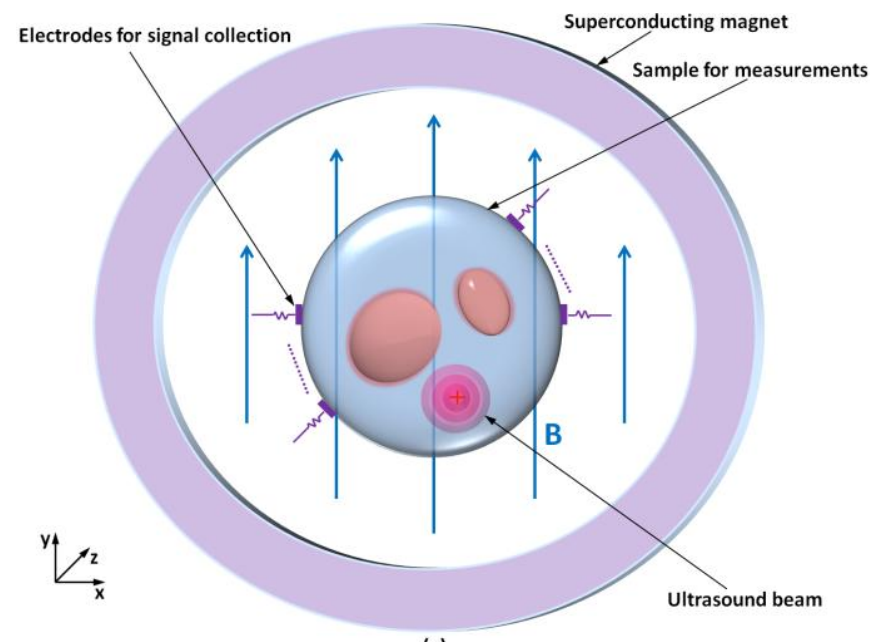

(c)

Fig. 1. (a) Configuration of permanent magnets based Halbach Array. (b) Configuration of HTS coils based Halbach Array. (c) Configuration of superconducting Lorentz Force Electrical Impedance Tomography (LFEIT).

weight and large geometry [2]. Therefore, the concept of superconducting Halbach Array magnet has been reasonably raised: High Temperature Superconducting (HTS) coils are placed as Halbach array configuration, which is demonstrated in Fig. 1 [2]. Superconducting Halbach array has a thin depth in $\mathrm{z}$ direction in Fig. 1 (a) and (b), which could potentially realise the portability for medical imaging devices, e.g. LFEIT working for emergency diagnosis in an ambulance [2]. Based on the previous results, the superconducting Halbach Array is able to achieve average magnetic flux density over $1 \mathrm{~T}$ and acceptable inhomogeneity within $60 \mathrm{~cm}$ diameter circular cross-section for a potential full-body LFEIT system [2]. 


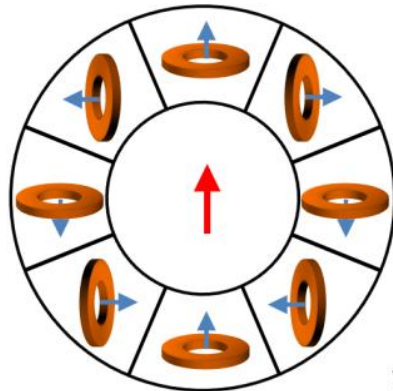

(a)
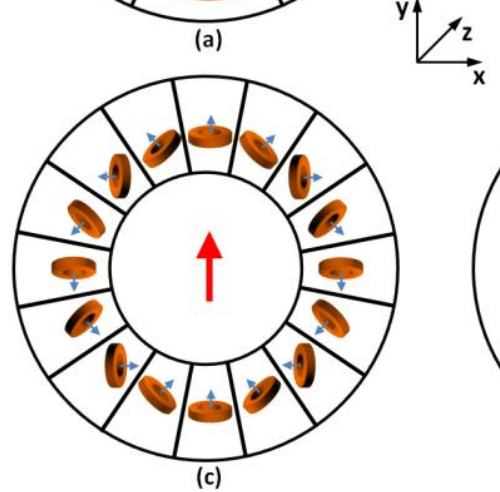

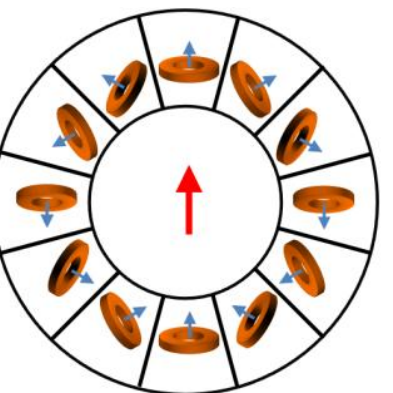

(b)

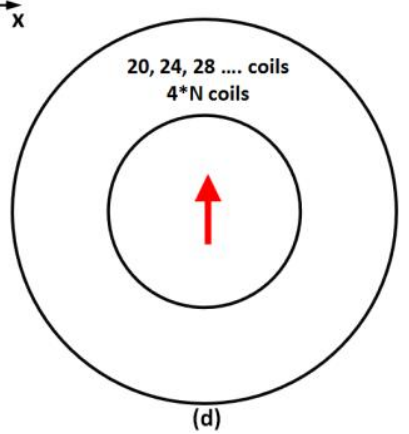

Fig. 2. Configuration of superconducting Halbach Array: (a) 8-coil, (b) 12-coil, (c) 16-coil, (d) increasing numbers of coils $4 \mathrm{~N}$ ( $\mathrm{N}$ is the integer starting from 2)

The quality of magnetic field, e.g. the strength and homogeneity, directly affect the imaging quality of biological tissues for LFEIT system [3, 5]. In our previous results, it has been deduced that the magnetic homogeneity can be improved by increasing the number of coils for Halbach arrangement while shrinking each coil's size, but overall without changing the total amount of superconductor [2].

To the best of our knowledge, there is no research on magnetic field optimization of superconducting magnet using the Halbach Array structure with HTS coils. This paper presents the further optimization on the coils' location and numbers of coils on the premise of maintaining the total amount of superconductor, to obtain the better homogeneity of magnetic field in the center area of superconducting Halbach Array. Furthermore, the mathematical expressions are derived for inhomogeneity estimation with the relation of different numbers (up to infinity) of HTS coils and distance to Halbach ring center.

\section{Modelling of superconducting Halbach}

\section{Array magnet}

\subsection{H-formulation}

To model the superconducting Halbach Array, 2D $\boldsymbol{H}$-formulation was used for the electromagnetic simulation, which calculates the solution of the induced current and magnetic field distribution on the basis on the (1) Faraday's Law, (2) Ampere's Law, (3) Ohm's Law, (4) Constitutive Law and (5) $E-J$ power law [6]:

$$
\nabla \times E=-\frac{\partial B}{\partial t}
$$

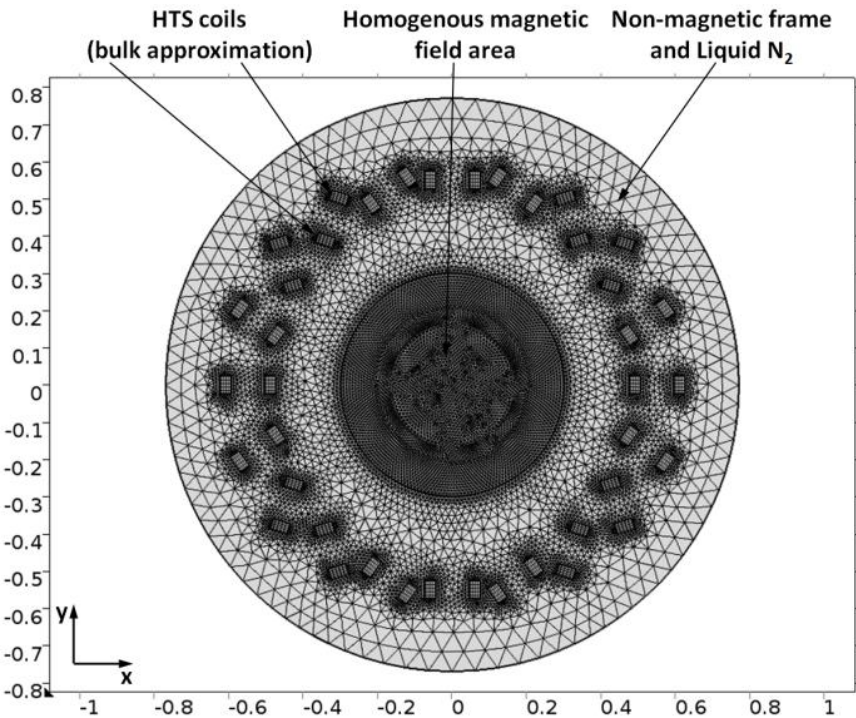

Fig. 3. Mesh for a 20-coil superconducting Halbach Array design.

Table 1 Specification for superconducting Halbach Array magnet

\begin{tabular}{ll}
\hline Parameters & Value \\
\hline Inner diameter & $60 \mathrm{~cm}$ \\
Outer diameter & $156 \mathrm{~cm}$ \\
$D_{c}$ (distance from coils center to & $55 \mathrm{~cm}$ \\
ring center) & \\
YBCO tape cross-section & $12 \mathrm{~mm} \times 0.1 \mathrm{~mm}$ \\
Bulk approximation cross-section & $48 \mathrm{~mm} \times$ ratio of turns \\
$\mu_{0}$ & $4 \pi \times 10^{-7} \mathrm{H} / \mathrm{m}$ \\
$n$ (E-J Power Law factor) & 21 \\
$J_{c 0}$ & $2.5 \times 10^{8} \mathrm{~A} / \mathrm{m}^{2}$ \\
$E_{0}$ & $10^{-4} \mathrm{~V} / \mathrm{m}$ \\
$I_{a p p}$ & $120 \mathrm{~A}$ \\
\hline
\end{tabular}

$$
\begin{gathered}
\nabla \times H=J \\
E=\rho J \\
B=\mu_{0} \mu_{r} H \\
E=E_{0}\left(\frac{J}{J_{c}}\right)^{n}
\end{gathered}
$$

where $\boldsymbol{E}$ is the electric field, $\boldsymbol{B}$ is the magnetic flux density, $\boldsymbol{H}$ is the magnetic field intensity, $\boldsymbol{J}$ is the current density, $\rho$ is the resistivity, $E_{0}$ is the characteristic electric field, $\mu_{0}$ is the permeability of free space, $\mu_{r}$ is the relative permeability, $J_{c}$ is the critical current density and $n$ is the factor for $E-J$ power law. By rearranging (1), (2), (3), (4) and (5), a general form of 2D partial differential equation (PDE) for dependent variables $\boldsymbol{H}$ can be computed by COMSOL Multiphysics:

$$
\frac{\partial\left(\mu_{0} \mu_{r} H\right)}{\partial t}+\nabla \times(\rho \nabla \times H)=0
$$

\subsection{Superconducting Halbach Arrray design}

Based on the Halbach Array ring configuration, the superconducting Halbach Array magnet was built using HTS coils. The modeling of superconducting Halbach was on the basis of the partial differential equation of $\boldsymbol{H}$-formulation in (6). The $12 \mathrm{~mm}$ HTS tape with critical $300 \mathrm{~A}$ at $77 \mathrm{~K}$, SuperPower SCS12050, was simulated as the coil material. As shown in Fig. 2 (a), (b) and (c), superconducting Halbach Array magnet consisted of 8,12 and 16 HTS coils, which 


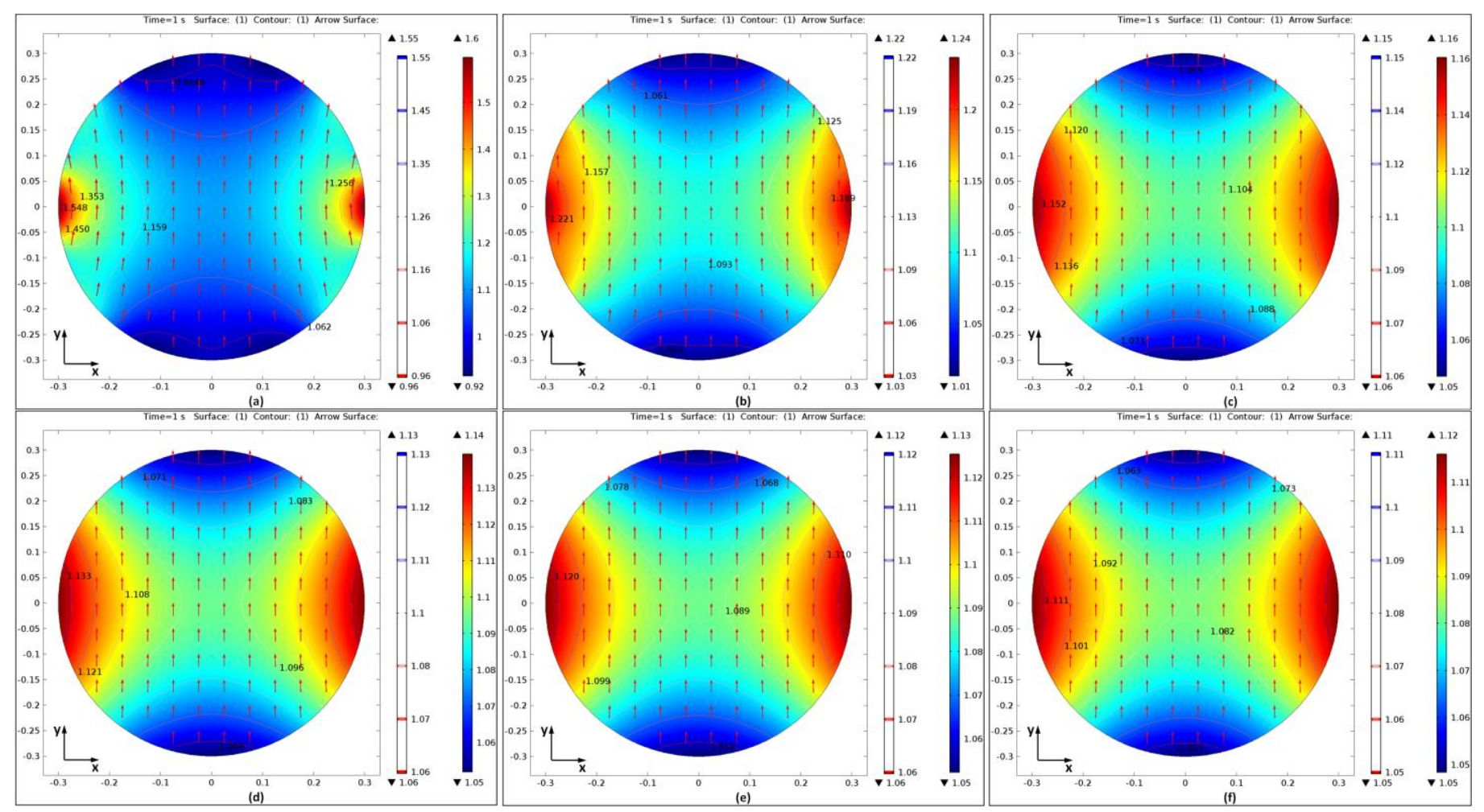

Fig. 4. Magnetic flux density (with direction and contour plot) in superconducting Halbach ring center cross-section: (a) 8-coil, (b) 12-coil, (c) 16-coil, (d) 20-coil, (e) 24-coil, (f) 28-coil.

followed the rule of $4 \mathrm{~N}$ ( $\mathrm{N}$ is the integer starting from 2) for the numbers of coil. Each coils carried the same amount of current. Each coil had a 360/2N degree phase change to the next coil, for example: 90 degree for 8-coil structure, 60 degree for 12-coil structure and 45 degree for 16-coil structure. The ideal direction of magnetic field generated from each coil is shown in Fig. 2. The specification of superconducting Halbach Array design is shown in Table 1. An example of mesh for 20-coil superconducting Halbach Array is illustrated in Fig. 3 (hidden air region outside the Halbach array was more than 100 times bigger).

As the critical current of HTS tape has the anisotropic characteristics, we added the $\boldsymbol{B}$-dependent critical current model to this design:

$$
J_{c}(B)=\frac{J_{c 0}}{\left(1+\sqrt{\frac{k^{2} B_{\text {para }}^{2}+B_{\text {perp }}^{2}}{B_{0}^{2}}}\right)}
$$

$J_{0}$ is the critical current density in self-field, $77 \mathrm{~K}$. Generally, the perpendicular component is much higher than the parallel component. In equation (7), $k=0.186$ and $B_{0}=0.426$ were chosen from previous work [2].

Similar to our previous study, stacked coils with different layers of substrate and normal materials were represented by continuous area bulk approximation [7, 8], which can efficiently improve simulation and speed model convergence $[9,10]$. The Physics function Pointwise constraint from general PDE model was used to inject transport current into the HTS coils, which forced the multiplication of the transport current $I_{s}$ with number of turns $\mathrm{N}$ equal to the integration of current density $J_{s}$ within bulk approximation over cross-section area $A$ :

$$
N I_{s}=\int J_{s} d A
$$

Each bulk approximation had the cross-section of $48 \mathrm{~mm}$ width, which represented 4 vertical stack coils $(4 \times 12 \mathrm{~mm})$. The bulk approximation thickness means the amount of turns, the higher thickness the more turns. Fig. 3 presents that the coils were fixed in the non-magnetic Halbach Array frame and within the liquid nitrogen at $77 \mathrm{~K}$. An overall DC current with $120 \mathrm{~A}$ for a single tape was applied using a ramp function to reach the peak current in the first 0.2 second and then maintained this DC value.

\section{Optimization and discussion}

The optimization is on the basis of without changing the total amount of superconductor, and increasing number of coils for Halbach arrangement by shrinking each coil's size.

Fig. 4 presents the most concerned part in this design, the 2D center cross-section of superconducting Halbach with six cases (a) 8-coil, (b) 12-coil, (c) 16-coil, (d) 20-coil, (e) 24-coil, (f) 28-coil structures. It can be seen that for all six cases the direction of magnetic flux is almost in $+y$ direction (the red arrow in Fig. 4), but the field direction is getting more normalized $+y$ direction when the number of coils increases from 8 to 28 , which is more obvious in the two end sides of $\mathrm{X}$-axis. According to the surface and contour plot in Fig. 4, the flux density is relatively uniform in the most part of the ring, but there are still two high-intensity areas in the end of the radius along x-axis. From colour bars of Fig. 4 (a) to (f), it can be seen that the difference between maximum and minimum of magnetic flux density $\left(B_{\max }-B_{\min }\right)$ decreases steadily from 


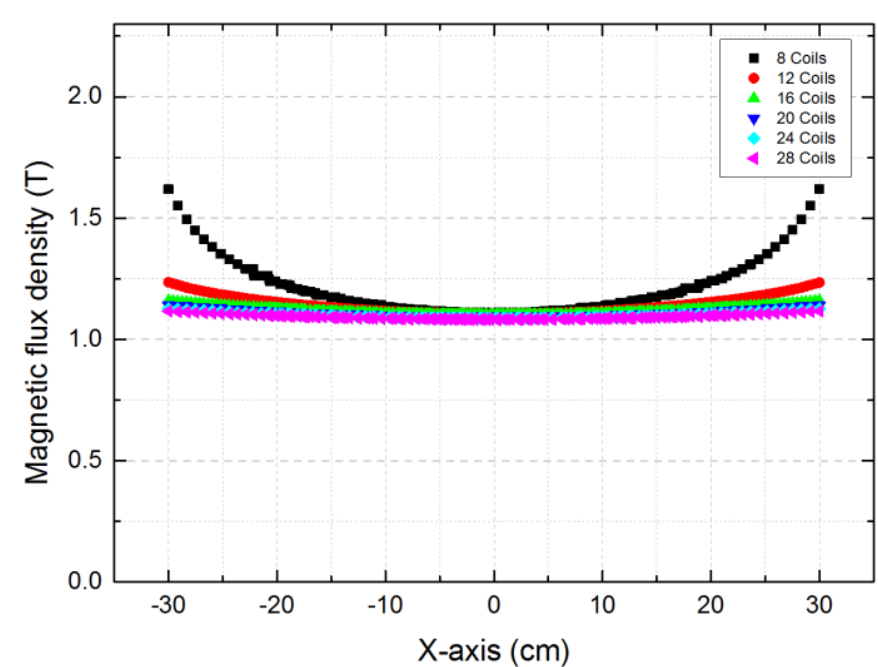

Fig. 5. Relationship between the magnetic flux density on diameter along $\mathrm{X}$-axis and different numbers of coils (six cases: 8, 12, 16, 20, 24 and 28 coils).

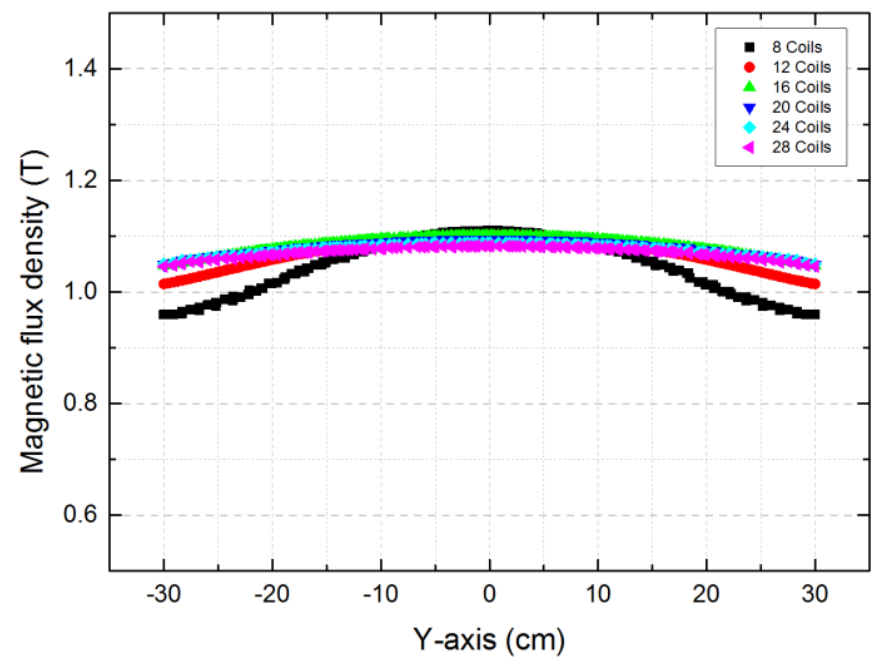

Fig. 6. Relationship between the magnetic flux density on diameter along Y-axis and different numbers of coils (six cases: 8, 12, 16, 20, 24 and 28 coils).

$0.68 \mathrm{~T}$ to $0.07 \mathrm{~T}$, which implies that the magnetic field in the cross-section becomes more homogeneous.

Fig. 5 illustrates the relationship between the magnetic flux density on diameter along $\mathrm{X}$-axis and different numbers of coils from 8 to 28 (six cases). From comparison, it can be discovered that in the end sides the 8-coil case has much higher magnetic flux density and it decreases sharply when the number of coils increases, while the middle parts of curves always have the similar value with all these six cases. Fig. 6 demonstrates the relationship between the magnetic flux density on diameter along $\mathrm{Y}$-axis and six cases different numbers of coils from 8 to 28 . With increasing the number of coils, both the end and the middle part of the curves become more and more flat, which could be clearly discovered from the comparison of 8-coil case (black curve) and the 28-case (purple curve). Overall, the inhomogeneity in both $\mathrm{X}$-axis and $\mathrm{Y}$-axis has been effectively reduced by this optimization method.

Precisely, Fig. 7 shows as relationship between surface (2D) inhomogeneity $\left(\left(B_{\max }-B_{\min }\right) / B_{\text {average }}\right)$ of magnetic flux density in the Halbach ring center cross-section and six cases for different numbers of coils. The vertical axis of Fig. 7 is in

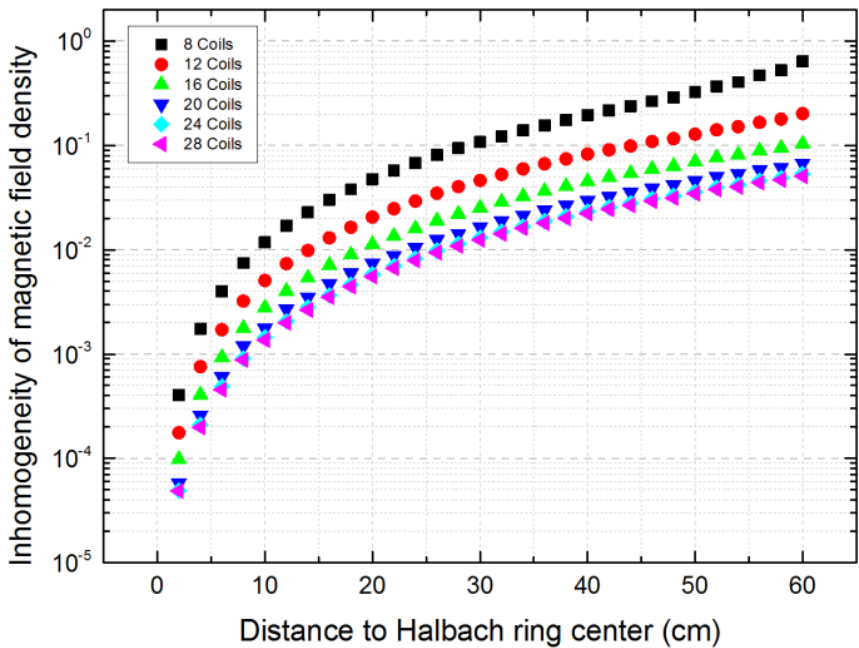

Fig. 7. Relationship between surface (2D) inhomogeneity of magnetic flux density in the Halbach ring center cross-section and different numbers of coils (six cases: 8, 12, 16, 20, 24 and 28 coils).

terms of log-scale. When the distance to Halbach ring center is less than $10 \mathrm{~cm}$, the 24-coil case and the 28-coil case have the inhomogenity in ppm (parts per million) level, which are less than $10^{-3}$. For the case of 28 coils, the inhomogeneity is below $5 \times 10^{-2}(50 \mathrm{ppk})$ in the entire region of Halbach ring center cross-section (60 cm diameter circular region). When distance to Halbach ring center is larger than $10 \mathrm{~cm}$, the inhomogenity of 28-coil case is approximately 1 order lower than the inhomogenity of 8-coil case. The magnetic homogeneity has improved significantly with the increasing number of coils but maintaining the same total amount of superconductor.

In order to further optimize the magnetic homogeneity in Halbach ring center cross-section, some mathematical relation between the inhomogeneity and increasing numbers of the coils could be investigated. The equation fitting results for the relation between the inhomogeneity, distance to Halbach ring center and numbers of the coils are:

$$
\begin{gathered}
\text { Inhomogeneity }=\alpha \cdot D^{\beta} \\
\alpha=a \cdot \exp (b \cdot n)+c \\
\beta=e \cdot \exp (f \cdot n)+g
\end{gathered}
$$

Equation (9) reveals that the inhomogeneity has the power law relation with the $D$ (distance to Halbach ring center). $\alpha$ and $\beta$ are the variables for power law which related to the $\mathrm{n}$ (numbers of coils) in equation (10) and (11). Both $\alpha$ and $\beta$ have exponential relation with $\mathrm{n}$ and adding a constant, where $a=1.408 \mathrm{E}-4, \quad b=-0.1556, \quad c=1.188 \mathrm{E}-05, \quad e=43.32$, $f=-0.5279, g=2.022$.

From equation (9), (10) and (11), it can be deduced that although homogeneity can be improved by the increasing numbers of the coils, the efficiency of homogeneity enhancement will be weakened when $n$ (numbers of coils) increase to high value. For instance, for $D=60 \mathrm{~cm}$, the inhomogeneity of 32-coil case is approximately one order smaller than 8-coil case, but from the mathematical estimation the inhomogenity of 64-coil case is only about $10 \%$ smaller than 32-coil case, and it is estimated that further increase of numbers of coils become much less efficient to reduce the inhomogenity. 
Therefore, it can be concluded that superconducting Halbach Array could potentially achieve homogeneous magnetic field in ppm level with the diameter less than 1/15 of its entire geometry (less than $10 \mathrm{~cm}$ distance to Halbach ring center), but it is difficult for superconducting Halbach Array to realise the magnetic inhomogeneity in ppm level in the entire region of Halbach ring center cross-section $(60 \mathrm{~cm}$ diameter circular region), with the method of increasing the number of coils while using the same total amount of superconductor. Another potential concern is these returning field lines associated with the actual thin depth could further slightly affect the inhomogeneity in the actual 3D geometry. However, for LFEIT system a compact superconducting Halbach Array is still usable, because 100 ppk inhomogeneity is sufficient owing to that LFEIT obtains the characteristic of ultrasound imaging to realise accurate location of signals and does not require highly homogeneous magnetic field if the magnetic strength is around $1 \mathrm{~T}$ [2, 5]. Halbach Array configuration based superconducting magnet could still be a feasible solution for uniform magnetic field while requiring relatively compact geometry.

\section{Conclusion}

The concept of Halbach Array structure based superconducting magnet has been proposed to generate an appropriate homogeneous magnetic field. The simulation has been executed on the FEM platform of COMSOL Multiphysics, which consists of 2D models of superconducting Halbach Array magnet using $\boldsymbol{H}$-formulation based on $\boldsymbol{B}$-dependent critical current density and bulk approximation. The optimization on the magnetic homogeneity has been carried out to increase the number of coils for Halbach arrangement while shrinking each coil's size, but still remain the total amount of superconductor. The mathematical relation between the inhomogeneity and increasing numbers of the coils has been derived using optimization results, which demonstrates that the magnetic homogeneity is able to be improved by the increasing the number of the coils, but the efficiency for homogeneity improvement will decrease when numbers of coils increase to high value. Overall, with this optimization method, Halbach Array configuration based superconducting magnet can potentially generate over $1 \mathrm{~T}$ uniform magnetic field with inhomogeneity in ppm level, which also has the advantage of relatively compact geometry. However, the fabrication difficulty and the optimization efficiency are needed to be taken into consideration.

\section{Acknowledgments}

The simulation work was carried out with the help of the Electrical Engineering Division, Department of Engineering, University of Cambridge. Authors are grateful to all members of staff for their useful assistance. Some of the authors are research students, and they would like to acknowledge China Scholarship Council (CSC) for their scholarships and support for overseas study.

\section{References}

[1] Y. Lvovsky, E. W. Stautner, and T. Zhang, "Novel technologies and configurations of superconducting magnets for MRI," Supercond. Sci. Technol., vol. 26, no. 9, pp. 093001, 2013.

[2] B. Shen, L. Fu, J. Geng, H. Zhang, X. Zhang, Z. Zhong, Z. Huang, and T. A. Coombs, "Design of a Superconducting Magnet for Lorentz Force Electrical Impedance Tomography," IEEE Trans. Appl. Supercond., vol. 26, no. 3, 2016.

[3] P. Grasland-Mongrain, J. Mari, J. Chapelon, and C. Lafon, "Lorentz force electrical impedance tomography," IRBM, vol. 34, no. 4, pp. 357360, 2013.

[4] K. Halbach, "Design of permanent multipole magnets with oriented rare earth cobalt material," Nucl. Instrum. Methods., vol. 169, no. 1, pp. 1-10, 1980.

[5] B. Shen, L. Fu, J. Geng, X. Zhang, H. Zhang, Q. Dong, C. Li, J. Li, and T. A. Coombs, "Design and simulation of superconducting Lorentz Force Electrical Impedance Tomography (LFEIT)," Phys. C, Supercond., vol. 524, pp. 5-12, 2016.

[6] Z. Hong, A. M. Campbell, and T. A. Coombs, "Numerical solution of critical state in superconductivity by finite element software," Supercond. Sci. Technol., vol. 19, no. 12, pp. 1246, 2006.

[7] V. M. Zermeno, A. B. Abrahamsen, N. Mijatovic, B. B. Jensen, and M. P. Sørensen, "Calculation of alternating current losses in stacks and coils made of second generation high temperature superconducting tapes for large scale applications," Journal of Applied Physics, vol. 114, no. 17, pp. 173901, 2013.

[8] J. Xia, H. Bai, J. Lu, A. V. Gavrilin, Y. Zhou, and H. W. Weijers, "Electromagnetic modeling of REBCO high field coils by the Hformulation," Superconductor Science and Technology, vol. 28, no. 12, pp. 125004, 2015.

[9] M. D. Ainslie, Y. Jiang, W. Xian, Z. Hong, W. Yuan, R. Pei, T. J. Flack, and T. A. Coombs, "Numerical analysis and finite element modelling of an HTS synchronous motor," Phys. C, Supercond., vol. 470, no. 20, pp. 1752-1755, 2010.

[10] L. Quéval, V. M. Zermeño, and F. Grilli, "Numerical models for ac loss calculation in large-scale applications of HTS coated conductors," Superconductor Science and Technology, vol. 29, no. 2, pp. 024007, 2016. 